\title{
Reduction of low- and high-grade cervical abnormalities associated with high uptake of the HPV bivalent vaccine in Scotland
}

\author{
K G J Pollock ${ }^{*}, 1,7, K_{\text {Kavanagh }}^{2,7}$, A Potts ${ }^{1}$, J Love ${ }^{1}$, K Cuschieri ${ }^{3}$, H Cubie $^{3}$, C Robertson $^{1}$, M Cruickshank ${ }^{4}$, \\ T J Palmer ${ }^{5}$, S Nicoll ${ }^{6}$ and M Donaghy ${ }^{1}$ \\ ${ }^{1}$ Health Protection Scotland, 5 Cadogan Street, Glasgow G2 60E, Scotland; ${ }^{2}$ Department of Mathematics and Statistics, University \\ of Strathclyde, Level 8, Livingstone Tower, 26 Richmond St, Glasgow G1 1XH, Scotland; ${ }^{3}$ Scottish Human Papillomavirus Reference \\ Laboratory, Royal Infirmary of Edinburgh, 51 Little France Crescent, Edinburgh EH16 4SA, Scotland; ${ }^{4}$ University of Aberdeen, \\ Health Sciences Building, Foresterhill, Aberdeen AB25 2ZD, Scotland; ${ }^{5}$ Raigmore Department of Pathology, Raigmore Hospital, \\ Old Perth Road, Inverness IV2 3UJ, Scotland and ${ }^{6}$ Department of Cytology, Ninewells Hospital, Dundee DD1 9SY, Scotland
}

Background: In Scotland, a national HPV immunisation programme began in 2008 for 12- to 13-year olds, with a catch-up campaign from 2008 to 2011 for those under the age of 18. To monitor the impact of HPV immunisation on cervical disease at the population level, a programme of national surveillance was established.

Methods: We analysed colposcopy data from a cohort of women born between 1988 and 1992 who entered the Scottish Cervical Screening Programme (SCSP) and were aged 20-21 in 2008-2012.

Results: By linking datasets from the SCSP and colposcopy services, we observed a significant reduction in diagnoses of cervical intraepithelial neoplasia 1 (CIN 1; RR 0.71, 95\% CI 0.58 to 0.87; P=0.0008), CIN 2 (RR 0.5, 95\% CI 0.4 to $0.63 ; P<0.0001$ ) and CIN 3 (RR $0.45,95 \% \mathrm{Cl} 0.35$ to $0.58 ; P<0.0001$ ) for women who received three doses of vaccine compared with unvaccinated women.

Conclusions: To our knowledge, this is one of the first studies to show a reduction of low- and high-grade CIN associated with high uptake of the HPV bivalent vaccine at the population level. These data are very encouraging for countries that have achieved high HPV vaccine uptake.

In the UK, cervical cancer is the second most common cancer in women under 35 years (Cancer research UK, 2010). Human papillomavirus (HPV) types 16 and 18 are known to be essential for the development of at least $70 \%$ of cervical cancers (Smith et al, 2007 ) but may contribute in excess of $80 \%$ of cervical cancers in Scotland (Cuschieri et al, 2010). The prophylactic bivalent vaccine prevents infection with HPV types 16 and 18 and has been shown to induce strong and sustained neutralising antibody responses that prevent cervical HPV 16 and 18 infection and confer protection against consequent viral-induced cervical intraepithelial neoplasia (CIN; Paavonen et al, 2009). The vaccine may also afford immunological cross-protection against other high-risk oncogenic HPV types that are phylogenetically related to HPV 16 and 18, including HPV 31, 33 and 45 (Malagon et al, 2012; Kavanagh et al, 2014).

The quadrivalent HPV vaccine has been provided in Australia through the national HPV vaccination programme since April 2007. Early indications from the HPV vaccination programme suggest that there has been a decrease in high-grade cervical cytological abnormalities (coded as CIN of grade 2 or worse or CGIN) of the cervix in girls younger than 18 years (Brotherton et al, 2011). Although the study did not directly link pathology

\footnotetext{
*Correspondence: Dr KGJ Pollock; E-mail: Kevin.pollock@nhs.net

${ }^{7}$ These authors contributed equally to this work.
}

Received 7 May 2014; revised 22 July 2014; accepted 7 August 2014; published online 2 September 2014 
results with immunisation status, it provided evidence that high uptake of the HPV vaccine ( $\sim 70 \%$ ) was temporally correlated with a decrease in cervical cancer precursors at the population level.

Since 2008, school-based uptake of bivalent HPV vaccine in girls aged 12-13 in Scotland has been impressive, with vaccine uptake sustained at levels $>90 \%$ (National Health Service (NHS) Information Services Division, 2011; Sinka et al, 2014). Furthermore, a 3-year (from September 2008 to 2011) catch-up campaign offered vaccination to all girls aged 13-17, with uptake recorded between $80 \%$ and $30 \%$ in younger and older girls at age of vaccination, respectively (NHS Information Services Division, 2012). In order to estimate vaccine impact it is important to ascertain the effect of the vaccination programme on the whole population, with particular focus on the age group where these changes will be initially observed. In Scotland cervical screening is offered 3-yearly to all women aged 20-60 years. Therefore it is one of the few countries in the world able to detect an early impact of the vaccine through population-based surveillance.

Scotland has a population of 5.2 million and almost all care is provided by the NHS. Preventive health programmes operate population registers based on birth and patient care registration systems with a common unique person identifier. One of the major strengths of Scottish health data is the ability to perform robust data linkage in a national population, using Community Health Index (CHI; Bhopal et al, 2012). In this study, we have used such linkage to complete preliminary analysis of the impact of the bivalent vaccine on $\mathrm{HPV}$-associated disease at the population level. These attributes allow us to demonstrate early impact of high HPV immunisation coverage to show significant reductions in the diagnosis of low- and high-grade CIN level.

\section{MATERIALS AND METHODS}

HPV surveillance cohorts. As part of the Health Protection Scotland (HPS) HPV surveillance strategy, cohorts of young women born between 1988 and 1992 were assessed to determine vaccine impact. The study identification number and the patient $\mathrm{CHI}$ reference (a unique national patient identifier) were sent to Information Services Division (ISD) of the NHS in Scotland, who used CHI to link the national Scottish Immunisation Recall System (SIRS) and Child Health Schools Programme-System (CHSP-S) data to screening and colposcopy attendance. This is described more elaborately elsewhere (Kavanagh et al, 2014).

The range of ages spans the period of eligibility for vaccination (1990-1992, i.e., the catch-up cohort) and also provides mainly unvaccinated individuals (1988-1989) from the early cohorts, for comparison. Geographical data-zone (Scottish Government, 2005) derived from the postcode of residence, was attributed to each record allowing assignment of the Scottish Index of Multiple Deprivation (SIMD; Scottish Government) to each individual in the cohorts.

Vaccination status derived from CHSP-S or the SIRS which act as the call and recall register for immunisation programmes in Scotland is linked to all individuals in the cohort.

Data linkage of the cohort. Women are referred directly by the Scottish Cervical Call and Recall System (SCCRS) to colposcopy for further investigation on the basis of high-grade dyskaryosis, repeat low-grade dyskaryosis or borderline nuclear abnormality. Colposcopy data are collected routinely for all referred individuals in NHS Scotland via the National Colposcopy Clinical Information and Audit System (NCCIAS). This information is episode based and comprises patient demographics, appointment details, clinical data including referral, clinical signs and symptoms, colposcopy assessment and findings, biopsy results, cytology results, treatments and the follow-up care management plan.
National Health Service ISD provided an extract of these data to HPS, matching NCCIAS patients to the HPV surveillance cohort via an anonymous reference number. Extracts from NCCIAS are received by HPS on a quarterly basis, with these data in this study based on linked referrals to the end of May 2013.

Statistical analysis. We restricted our analysis to those individuals in the cohort with a cervical screening attendance date in SCCRS after the age of eligibility (age 20). In the NCCIAS dataset, individuals may have more than one linked histological episode to the index referral cytology. We restricted our analysis to the incident abnormal histological episode. Incidence rates per 1000 person-year (p1000py) were calculated by comparing the number of cases of each diagnosis to the number of individuals screened, adjusting for the person-time contribution of each individual as those born in the early cohorts have more time to develop an outcome. This contribution was calculated as the number of months between the individual attending for their first screen and the date of referral for the incident abnormal histological episode if present (which could occur following a later screening attendance, or alternatively to 31st May 2013 (the latest month for which referrals were extracted), whichever occurred first. Incidence rates p1000py were stratified by vaccination status and birth cohort.

The relative risk of CIN in the vaccinated population compared to the unvaccinated population was calculated using Poisson regression adjusting for cohort year to model potential changes in sociological behaviour that may exist from one birth cohort to the next, deprivation score (assessed via the SIMD quintiles of the area of residence where 1 is most deprived and 5 is least deprived) and age. Person-time contribution was used as an offset. Individuals were censored at the date of referral for an incident abnormal histological episode (all grades) or on 31st May 2013. As the risk of an abnormal histological episode may change over time, age is included as a time-dependent covariate, with person-time contribution and the number of abnormal histological episodes stratified by the age of the individual in months as they move through the study. For each grade of CIN, interaction tests were carried out to consider differences in the vaccine effect between cohort years and between deprivation (SIMD quintile).

As a sensitivity analysis, we considered only the abnormal histological episodes that followed the first cervical screen in only those who attended for screening at age 20 or 21 . The odds of CIN in the vaccinated population compared to the unvaccinated population were then calculated using logistic regression adjusting for cohort year and deprivation score for grades CIN 1, 2 and 3 individually. This analysis considers a more homogeneous population in terms of age and attendance for screening, and age at histological examination. All statistical analysis was conducted in $\mathrm{R}$ version 3.0.3 (Vienna, Austria) (R Development Core Team, 2014).

\section{RESULTS}

Cohort analysis. Across all five birth cohorts $(n=200867), 53.5 \%$ $(n=106052)$ attended for their first cervical screen at age 20 or above (Table 1). Attendance was lowest for the youngest cohort, that is, those born in 1992 (36.3\%) with these individuals having less follow-up time at screening attendance, at the time of data extraction. Across all five cohorts, $72 \%$ were unvaccinated and $24 \%$ received three doses of vaccination, with vaccine uptake varying significantly by cohort year. Ninety-nine percent of those born in 1988-1989 were unvaccinated since the programme was not targeted at these individuals. A proportion of those born in 1990 and all of those born in 1991-1992 were eligible for vaccination. Vaccine uptake increased yearly and was highest for the 1992 cohort where $74 \%$ of individuals received three doses (Table 1). 
The first result for an abnormal referral (this includes a result of CIN 1 or worse) occurring after the date of first screen for each individual was considered. Those with a date of referral prior to the date of first screen were excluded $(n=10)$, reducing the total cohort size from 106052 to 106042 . Of the 10 excluded individuals, 5 individuals had CIN 1 and were all unvaccinated, four individuals had CIN 2, three of whom were unvaccinated and one individual had CIN 3 who was unvaccinated. In total there were 4854 abnormal histology (CIN 1-3) episodes; 1753 were CIN 1, 1698 CIN 2 and 1403 CIN 3 (Table 1).

Incidence and relative risk of $\mathrm{CIN} 1,2$ and 3 . Incidence rates for CIN 1, 2 and 3 diagnosis p1000py by number of vaccinations received and birth cohort were estimated (Figure 1). For those fully vaccinated in 1990-1992, there is a clear reduction in incidence of CIN 3 between unvaccinated and fully vaccinated individuals, for example, in 1991, the incidence in the unvaccinated is 7.93 p1000py $(95 \%$ CI $6.13,10.10)$ compared to 3.66 p1000py $(95 \%$ CI $2.80,4.69)$ in those receiving three doses. This reduction in incidence of CIN 3 was statistically significant in both the unadjusted and adjusted models (three dose unadjusted, RR 0.59, 95\% CI 0.48, 0.72; $P<0.0001$; and three dose adjusted, RR 0.45, $95 \%$ CI $0.35,0.58 ; P<0.0001$; Table 2). Although those receiving two doses of vaccine had a lower incidence rate of CIN 3 than the unvaccinated group in the 1990-1992 cohorts, the adjusted relative risk was not statistically significant (two dose adjusted, RR 0.77 , 95\% CI $0.49,1.21 ; P=0.25$ ).

The adjusted analysis (Table 2) also showed a statistically significant difference in relative risk of diagnoses of CIN 2 (RR 0.5 , 95\% CI $0.4,0.63 ; P<0.0001$ ) and CIN 1 (RR 0.71, 95\% CI $0.58,0.870 ; P=0.0008)$ associated with three doses of vaccine compared with those who were unvaccinated. Two doses of vaccine were associated with a reduced risk of both CIN 2 and CIN 1 but this was not statistically significant (CIN 2: RR 0.81 , $95 \%$ CI $0.54,1.22 ; P=0.32$ and CIN 1: RR 0.65, 95\% CI 0.42, 1.01; $P=0.056)$.

Relative risk of CIN 1, 2 and 3 diagnosis was significantly lower for the least deprived women (SIMD 5) compared to the most deprived (SIMD 1), even when differences in vaccination were accounted for (Table 2). For each outcome, the relative risk of a diagnosis was significantly lower among women from affluent areas compared to women from very deprived areas.

Table 2 also shows that there was a significant reduction in all grades of CIN in those born in 1989. Furthermore, for CIN 3 there are significant reductions in those born in the 1989 and 1992 cohorts but not in those born in 1990 and 1991. Generally the trend is downwards and conducting a test for linear trend shows that for each of CIN 1, 2 and 3 there is a significant linear change over the cohort years $(P=0.0126, P<0.0001$ and $P=0.0009$, respectively).

Table 1. Breakdown of each birth cohort in terms of number screened and the proportion vaccinated against HPV 16 and 18 ( $n=106052)$ and the number of cervical abnormalities (CIN 1-3) found in the follow-up period (to 31 st May 2013)

\begin{tabular}{|c|c|c|c|c|c|c|c|c|c|c|}
\hline \multirow[b]{2}{*}{$\begin{array}{l}\text { Cohort } \\
\text { year }\end{array}$} & \multicolumn{3}{|c|}{$\begin{array}{c}\text { Attendance at cervical screening at } \\
\text { age } \geqslant 20\end{array}$} & \multicolumn{4}{|c|}{$\begin{array}{l}\% \text { of screened population } \\
\text { immunised }\end{array}$} & \multicolumn{3}{|c|}{$\begin{array}{l}\text { Number of cervical abnormalities } \\
\text { among those screened }\end{array}$} \\
\hline & $\begin{array}{l}\text { Screened } \\
(N)\end{array}$ & Total $(N)$ & $\begin{array}{c}\% \\
\text { screened }\end{array}$ & $\begin{array}{l}\text { Unvaccinated } \\
(\%)\end{array}$ & $\begin{array}{l}1 \text { Dose } \\
\text { (\%) }\end{array}$ & $\begin{array}{l}2 \text { Doses } \\
(\%)\end{array}$ & $\begin{array}{l}3 \text { Doses } \\
(\%)\end{array}$ & CIN 1 & CIN 2 & $\mathrm{CIN} 3$ \\
\hline 1988 & 26021 & 41948 & 62.00 & 99.95 & 0.01 & 0.01 & 0.03 & 638 & 670 & 559 \\
\hline 1989 & 22168 & 40618 & 54.60 & 99.66 & 0.12 & 0.07 & 0.15 & 449 & 474 & 378 \\
\hline 1990 & 23124 & 39377 & 58.72 & 82.29 & 1.41 & 2.63 & 13.67 & 375 & 320 & 276 \\
\hline 1991 & 20510 & 39672 & 51.70 & 31.03 & 3.02 & 6.70 & 59.24 & 217 & 175 & 151 \\
\hline 1992 & 14229 & 39252 & 36.30 & 18.45 & 2.41 & 5.06 & 74.08 & 74 & 59 & 39 \\
\hline Total & $106052^{\mathrm{a}}$ & 200867 & 52.70 & 71.77 & 1.24 & 2.57 & 24.42 & 1753 & 1698 & 1403 \\
\hline
\end{tabular}
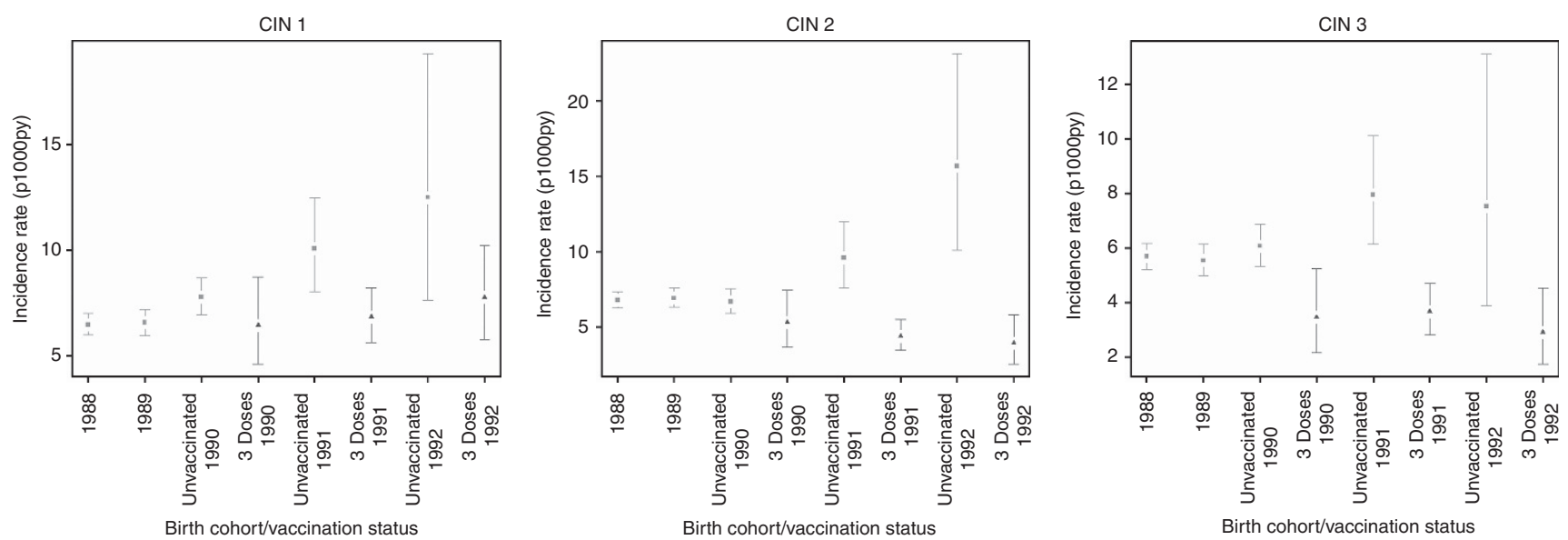

Figure 1. Incidence rates per 1000 person-year (p1000py) and associated 95\% confidence intervals of CIN 1, 2 and 3 stratified by birth cohort and vaccination status (unvaccinated vs fully vaccinated with three doses). 


\begin{tabular}{|c|c|c|c|c|c|c|c|c|c|c|c|c|c|c|c|c|c|}
\hline \multirow{3}{*}{ 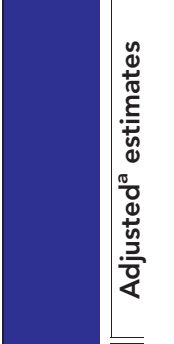 } & $\frac{\frac{0}{2}}{\frac{\pi}{\pi}}$ & & & $\mid \begin{array}{l}\frac{l}{f} \\
\frac{f}{0} \\
\end{array}$ & $\stackrel{\stackrel{n}{0}}{\circ}$ & $\begin{array}{l}\bar{o} \\
\text { o } \\
\dot{0} \\
v\end{array}$ & & $\begin{array}{l}\infty \\
0 \\
0 \\
0 \\
0\end{array}$ & 足 & 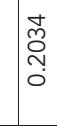 & õ & & 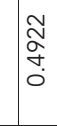 & $\begin{array}{l}\bar{\delta} \\
\grave{o} \\
\dot{0}\end{array}$ & 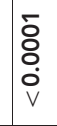 & $\begin{array}{l}\bar{\delta} \\
\text { ò } \\
\dot{0} \\
v\end{array}$ & \\
\hline & $\begin{array}{l}\bar{U} \\
\text { ㅇํㅇ }\end{array}$ & & & \begin{tabular}{|l}
$\infty$ \\
$\stackrel{\infty}{1}$ \\
$\sim$ \\
$\infty$ \\
$\infty$ \\
0 \\
0
\end{tabular} & 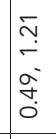 & $\begin{array}{l}\infty \\
0 \\
0 \\
0 \\
0 \\
\infty \\
0 \\
0 \\
0\end{array}$ & I & $\begin{array}{l}0 \\
0 \\
0 \\
0 \\
0 \\
0 \\
0 \\
0\end{array}$ & $\mid \begin{array}{c}1 \\
0 \\
\vdots \\
0 \\
0 \\
0 \\
0\end{array}$ & {$\left[\begin{array}{l}\infty \\
0 \\
\hdashline \\
\infty \\
\infty \\
0 \\
0\end{array}\right.$} & $\begin{array}{l}\tilde{\hat{\lambda}} \\
0 \\
0 \\
\hat{j} \\
0 \\
0\end{array}$ & & 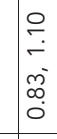 & $\begin{array}{c}\hat{n} \\
0 \\
0 \\
0 \\
0 \\
0 \\
0\end{array}$ & $\begin{array}{l}m \\
\tilde{n} \\
0 \\
\tilde{N} \\
\tilde{n} \\
0\end{array}$ & $\begin{array}{l}\hat{n} \\
0 \\
0 \\
0 \\
0 \\
0 \\
0\end{array}$ & \\
\hline & $\frac{\alpha}{\alpha \underline{x}}$ & & - & $\stackrel{\mathcal{Z}}{\leftarrow}$ & $\underset{0}{\hat{0}}$ & 佲 & - & $\begin{array}{l}+ \\
\infty \\
0 \\
0\end{array}$ & $\begin{array}{l}\infty \\
\infty \\
0 \\
0\end{array}$ & {$\left[\begin{array}{l}\infty \\
\infty \\
0\end{array}\right.$} & fo & - & 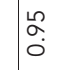 & 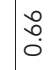 & ¿. & f̊. & \\
\hline \multirow{4}{*}{ 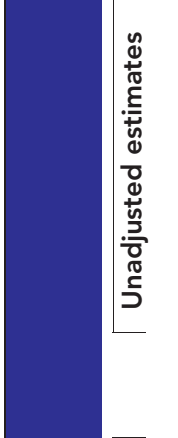 } & $\frac{\frac{0}{2}}{\frac{\pi}{\pi}}$ & & & 它 & 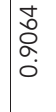 & $\begin{array}{l}\bar{\delta} \\
\dot{0} \\
\dot{0}\end{array}$ & & 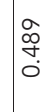 & $\begin{array}{l}\widetilde{N} \\
\widetilde{\alpha} \\
\alpha\end{array}$ & {$\left[\begin{array}{l}\infty \\
0 \\
0 \\
0\end{array}\right.$} & $\underset{\substack{m \\
c}}{c}$ & & 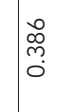 & $\begin{array}{l}\bar{\delta} \\
\grave{o} \\
\dot{0} \\
v\end{array}$ & $\begin{array}{l}\bar{\delta} \\
\grave{o} \\
\dot{0} \\
v\end{array}$ & $\begin{array}{l}\bar{\delta} \\
\text { ò } \\
\dot{0} \\
v\end{array}$ & \\
\hline & 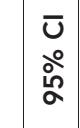 & & & 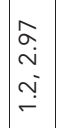 & 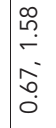 & $\begin{array}{l}\hat{N} \\
0 \\
0 \\
o ̛ ̀ \\
o ̛ ⿱ \\
0\end{array}$ & & 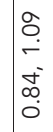 & $\mid \begin{array}{c}0 \\
\\
\\
0 \\
0 \\
0\end{array}$ & 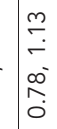 & 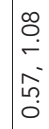 & & $\begin{array}{l}\infty \\
\stackrel{\infty}{c} \\
0 \\
\infty \\
0 \\
0\end{array}$ & 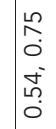 & $\begin{array}{l}\hat{n} \\
\hat{0} \\
\omega \\
0 \\
0\end{array}$ & 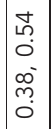 & \\
\hline & $\frac{\alpha}{\alpha}$ & & - & $\begin{array}{c}\stackrel{a}{\infty} \\
\stackrel{-}{-}\end{array}$ & $\stackrel{\infty}{\stackrel{\infty}{-}}$ & 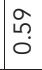 & & $\begin{array}{l}\text { L2 } \\
\text { o. }\end{array}$ & $\underset{-}{\sigma}$ & o. & 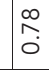 & & da & $\begin{array}{l}\text { f } \\
0 \\
0\end{array}$ & 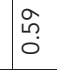 & 告 & \\
\hline & & $\frac{m}{\underline{z}}$ & & & & & & & & & & & & & & & \\
\hline \multirow{3}{*}{ 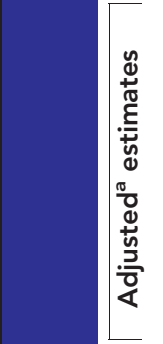 } & 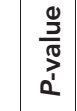 & & & \begin{tabular}{|c|}
$\tilde{\alpha}$ \\
$\infty$ \\
$\alpha$ \\
$\alpha$ \\
0
\end{tabular} & 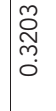 & $\begin{array}{l}\bar{b} \\
\grave{o} \\
\dot{v} \\
\mathrm{v}\end{array}$ & & $\begin{array}{l}\hat{0} \\
\frac{0}{0} \\
0\end{array}$ & oُ & $\left\{\begin{array}{l}\tilde{N} \\
0 \\
0 \\
0\end{array}\right.$ & ¿̊ & & $\begin{array}{l}\overline{1} \\
\text { ơ } \\
0\end{array}$ & $\begin{array}{l}\bar{\delta} \\
\grave{o} \\
\dot{0} \\
\mathrm{v}\end{array}$ & $\begin{array}{l}\bar{o} \\
\grave{o} \\
\dot{0} \\
v\end{array}$ & $\begin{array}{l}\bar{\delta} \\
\text { ó } \\
\dot{0} \\
\text { v }\end{array}$ & \\
\hline & $\begin{array}{l}\bar{U} \\
\text { ㅇํㅇ } \\
\text { 잉 }\end{array}$ & & & 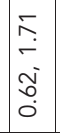 & 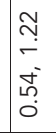 & $\begin{array}{l}3 \\
0 \\
0 \\
0 \\
0 \\
0 \\
0 \\
0\end{array}$ & I & $\begin{array}{l}\hat{a} \\
0 \\
0 \\
0 \\
\hat{o} \\
0\end{array}$ & $\mid \begin{array}{l}2 \\
0 \\
0 \\
0 \\
0 \\
0 \\
0\end{array}$ & 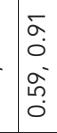 & $\begin{array}{l}0 \\
0 \\
0 \\
0 \\
0 \\
0 \\
0 \\
0\end{array}$ & I & $\begin{array}{l}2 \\
\alpha \\
0 \\
0 \\
0 \\
0 \\
0\end{array}$ & $\begin{array}{l}0 \\
0 \\
0 \\
0 \\
0 \\
0 \\
0 \\
0\end{array}$ & 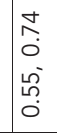 & $\begin{array}{l}\text { م⿱⺊口 } \\
0 \\
0 \\
o ̛ ⿱ \\
0 \\
0 \\
0\end{array}$ & 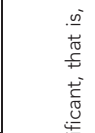 \\
\hline & $\frac{\alpha}{\alpha}$ & & - & 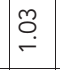 & $\begin{array}{l}\bar{\infty} \\
0 \\
0\end{array}$ & مُ & - & 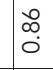 & $\begin{array}{l}\infty \\
\stackrel{0}{0}\end{array}$ & 前 & 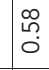 & - & 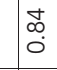 & $\begin{array}{l}0 \\
0 \\
0\end{array}$ & 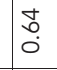 & f̊. & \\
\hline \multirow{4}{*}{ 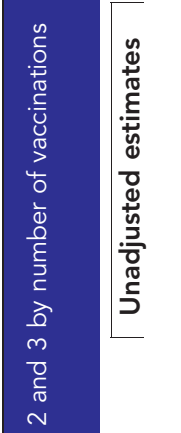 } & $\frac{0}{\frac{0}{3}}$ & & & 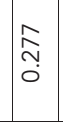 & $\stackrel{\infty}{\infty}$ & $\begin{array}{l}\bar{\delta} \\
\varnothing \\
\dot{0} \\
\text { v }\end{array}$ & & $\mid \begin{array}{c}\mathbb{N} \\
\infty \\
0 \\
0\end{array}$ & $\begin{array}{l}\alpha \\
\hat{f} \\
\sigma\end{array}$ & {$\left[\begin{array}{l}\infty \\
\infty \\
\sigma\end{array}\right.$} & $\begin{array}{l}\tilde{\sigma} \\
\delta \\
0 \\
0\end{array}$ & & 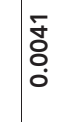 & $\begin{array}{l}\bar{\delta} \\
\text { ò } \\
\dot{0} \\
\mathrm{v}\end{array}$ & $\begin{array}{l}\bar{o} \\
\grave{o} \\
\dot{0} \\
v\end{array}$ & $\begin{array}{l}\bar{\delta} \\
\grave{o} \\
\dot{0} \\
\text { v }\end{array}$ & 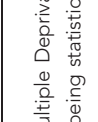 \\
\hline & $\begin{array}{l}\bar{U} \\
\stackrel{\circ}{\circ} \\
\text { 응 }\end{array}$ & & & 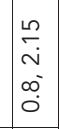 & 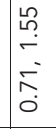 & 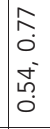 & & 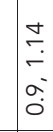 & 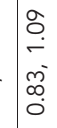 & $\left\{\begin{array}{l}0 \\
\stackrel{0}{c} \\
\stackrel{2}{2} \\
0\end{array}\right.$ & 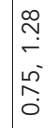 & & $\begin{array}{l}0 \\
0 \\
0 \\
0 \\
0 \\
0 \\
0 \\
0\end{array}$ & $\begin{array}{l}\infty \\
0 \\
0 \\
0 \\
\infty \\
0 \\
0 \\
0\end{array}$ & $\begin{array}{l}\tilde{N} \\
\tilde{N} \\
\tilde{N} \\
\tilde{n} \\
0 \\
0\end{array}$ & $\begin{array}{l}\tilde{n} \\
0 \\
0 \\
\alpha \\
0 \\
m \\
0\end{array}$ & 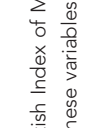 \\
\hline & $\frac{\mathscr{\alpha}}{\alpha}$ & & - & 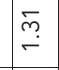 & $\stackrel{\leftrightarrow}{\circ}$ & 吾 & & $\underset{-}{\bar{\sigma}}$ & $\begin{array}{l}\stackrel{2}{\alpha} \\
0 \\
0\end{array}$ & {$\left[\begin{array}{l}a \\
\infty \\
0 \\
0\end{array}\right.$} & $\mid \begin{array}{l}\infty \\
0 \\
0 \\
0\end{array}$ & & $\stackrel{\substack{\infty \\
0 \\
\infty}}{0}$ & 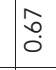 & Õ. & 年 & \\
\hline & & $\begin{array}{l}N \\
Z \\
U\end{array}$ & & & & & & & & & & & & & & & 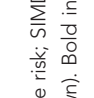 \\
\hline \multirow{3}{*}{ 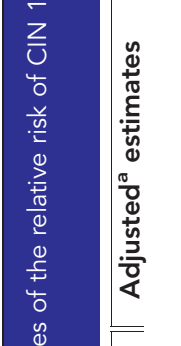 } & 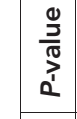 & & & $\begin{array}{l}\bar{\sigma} \\
\sigma \\
\sigma \\
o \\
o\end{array}$ & $\begin{array}{l}\hat{n} \\
\text { od } \\
\text { o. }\end{array}$ & 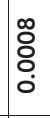 & & $\begin{array}{l}\text { D } \\
\vdots \\
\vdots \\
0 \\
0\end{array}$ & $\begin{array}{l}\tilde{N} \\
\tilde{D} \\
\tilde{N} \\
0\end{array}$ & 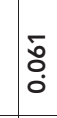 & 苋 & & $\frac{\mathfrak{N}}{\mathfrak{N}}$ & $\frac{0}{0}$ & ঃั & 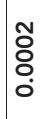 & \\
\hline & $\begin{array}{l}\bar{U} \\
\stackrel{\circ}{\circ} \\
\text { 응 }\end{array}$ & & & 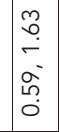 & 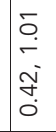 & $\begin{array}{l}\infty \\
\infty \\
0 \\
\infty \\
\infty \\
0 \\
0 \\
0 \\
0\end{array}$ & I & $\begin{array}{l}\hat{a} \\
0 \\
0 \\
0 \\
o \\
o\end{array}$ & $\mid \begin{array}{c}\hat{0} \\
\dot{\alpha} \\
\dot{\alpha} \\
\infty \\
\infty \\
0\end{array}$ & $\mid \begin{array}{c}\bar{\sigma} \\
\dot{\alpha} \\
\hat{o} \\
0 \\
0\end{array}$ & $\begin{array}{l}0 \\
0 \\
0 \\
0 \\
0 \\
0 \\
0 \\
0\end{array}$ & I & $\begin{array}{c}0 \\
2 \\
0 \\
0 \\
0 \\
0 \\
0\end{array}$ & $\begin{array}{l}\hat{\alpha} \\
0 \\
\hat{m} \\
\hat{o} \\
\hat{o}\end{array}$ & $\begin{array}{l}\text { a } \\
0 \\
0 \\
0 \\
0 \\
0 \\
0 \\
0\end{array}$ & $\begin{array}{l}\infty \\
\infty \\
0 \\
0 \\
0 \\
0 \\
0 \\
0 \\
0\end{array}$ & 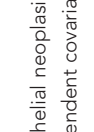 \\
\hline & $\stackrel{\alpha}{\alpha}$ & & - & $\begin{array}{l}\infty \\
0 \\
0 \\
0\end{array}$ & $\begin{array}{l}\text { Ln } \\
0 \\
0 \\
0\end{array}$ & î & - & $\begin{array}{l}\circ \\
\infty \\
0 \\
0\end{array}$ & o. & {$\left[\begin{array}{l}\infty \\
\infty \\
0 \\
0\end{array}\right.$} & $\begin{array}{l}0 \\
0 \\
0 \\
0\end{array}$ & - & {$\left[\begin{array}{l}t \\
0 \\
0 \\
0\end{array}\right.$} & $\begin{array}{l}\text { a } \\
0 \\
0 \\
0\end{array}$ & $\begin{array}{c}\infty \\
\infty \\
0 \\
0\end{array}$ & $\stackrel{0}{\stackrel{0}{0}}$ & \\
\hline \multirow{4}{*}{ 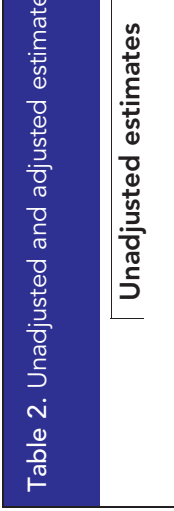 } & 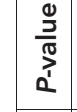 & & & 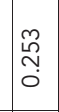 & $\begin{array}{l}\tilde{N} \\
\tilde{D} \\
0 \\
0\end{array}$ & 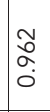 & & 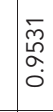 & 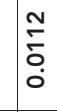 & $\begin{array}{l}0 \\
\hat{o} \\
0 \\
0 \\
0\end{array}$ & $\begin{array}{l}\text { o̊ } \\
\text { o̊ } \\
0\end{array}$ & & $\begin{array}{l}\hat{\alpha} \\
0 \\
0 \\
0\end{array}$ & $\bar{\delta}$ & 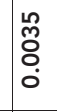 & סे & \\
\hline & 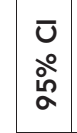 & & & $\begin{array}{l}\infty \\
\\
\dot{\infty} \\
\infty \\
0 \\
0\end{array}$ & 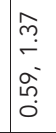 & $\begin{array}{l}0 \\
\stackrel{0}{0} \\
0 \\
0 \\
0 \\
0\end{array}$ & | & $\mid \begin{array}{c}\stackrel{m}{c} \\
\vec{c} \\
\alpha \\
0 \\
0 \\
0\end{array}$ & 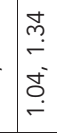 & 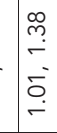 & 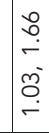 & I & 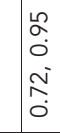 & $\begin{array}{l}0 \\
0 \\
0 \\
0 \\
0 \\
0 \\
0 \\
0\end{array}$ & $\begin{array}{l}m \\
2 \\
0 \\
0 \\
0 \\
0 \\
0 \\
0\end{array}$ & $\begin{array}{l}0 \\
0 \\
0 \\
0 \\
0 \\
0 \\
0 \\
0 \\
0\end{array}$ & 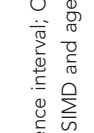 \\
\hline & $\stackrel{\propto}{\propto}$ & & - & $\stackrel{m}{\stackrel{m}{-}}$ & $\stackrel{\circ}{\circ}$ & - & - & - & $\stackrel{\infty}{\stackrel{\infty}{\leftarrow}}$ & $\stackrel{\infty}{\check{r}}$ & $\stackrel{m}{-}$ & - & {$\left[\begin{array}{c}\infty \\
0 \\
0\end{array}\right.$} & $\begin{array}{l}\infty \\
\infty \\
0 \\
0\end{array}$ & $\stackrel{\infty}{\infty}$ & $\stackrel{i}{\stackrel{5}{0}}$ & \\
\hline & & $\bar{z}$ & 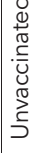 & $\begin{array}{l}\stackrel{d}{0} \\
\stackrel{\Delta}{\Delta} \\
-\end{array}$ & 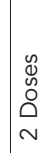 & 品 & & & $\underset{\stackrel{\circ}{\circ}}{\stackrel{\circ}{\sigma}}$ & $\underset{\sigma}{\alpha}$ & $\begin{array}{l}\alpha \\
\alpha \\
\sigma\end{array}$ & $\sum_{i}^{\bar{i}}$ & $\sum_{i=}^{N}$ & $\sum_{i}^{m}$ & $\sum_{i}^{+}$ & $\sum_{\substack{n \\
n}}^{n}$ & 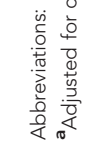 \\
\hline
\end{tabular}


There was neither significant interaction between the number of vaccine doses and deprivation (SIMD score) on occurrence of CIN 1,2 or 3 ( $P$-value of interaction tests $P=0.65, P=0.811$ and $P=0.63$, respectively) nor between the number of vaccine doses and the birth cohorts ( $P$-value of interaction tests $P=0.48, P=0.1$ and $P=0.86$, respectively).

Sensitivity analysis. We conducted a sensitivity analysis of only those individuals screened at age 20 or 21 and consider only incident abnormal histology occurring within 6 months of their first screen. This reduced our total cohort size to 91677 of which $73 \%$ were unvaccinated and $23 \%$ fully vaccinated. Within this reduced cohort, there were 602 CIN 1, 617 CIN 2 and 575 CIN 3 diagnoses. In adjusted analyses, women vaccinated with three doses had a statistically significant reduced risk of being diagnosed with CIN 1, 2 and 3 (Table 3), similar to that observed in the ageadjusted model (Table 2) although the confidence intervals were wider due to the smaller cohort size. As in the full model, two doses of vaccine was associated with a reduction in each of CIN 1, 2 and 3 but this was not statistically significant $(P=0.176, P=0.121$ and $P=0.352$, respectively).

In this analysis, there was no significant change in the numbers of CIN 1 and 2 by cohort year ( $P$-values of the linear test of trend were 0.497 and 0.4463 , respectively). For CIN 3, there was evidence of a linear trend $(P=0.044)$ driven by the large reduction in the 1992 cohort coupled with marginal reductions in the preceding birth cohorts.

\section{DISCUSSION}

In this study, we have completed a preliminary analysis of the impact of the bivalent vaccine on HPV-associated cervical disease at the population level. To our knowledge, this is the first population-based study to report a statistically significant decrease in incidence of cervical intraepithelial neoplasia grades 1,2 and 3 (29\%, 50\% and 55\%, respectively) in women aged $20-21$, associated with three doses of bivalent HPV vaccine administered during a catch-up campaign.
The vaccinated women in this cohort received the HPV vaccine through a national catch-up campaign, with mean uptake across all age groups for all three doses recorded as 66\% (NHS Information Services Division, 2012). Since 2008, the uptake rate of the HPV vaccine in the routine cohort (immunised at age $12-13$ years) has consistently achieved $>90 \%$ (NHS Information Services Division, 2011) and therefore any effect on HPV-associated disease is likely to be even more profound than that observed in the catch-up cohort. These data are very encouraging for countries that have achieved high HPV vaccine uptake.

Long-term surveillance of the effects of HPV vaccination in the population commenced in Scotland in 2008 and incorporates the study of both HPV prevalence in residual liquid-based cytology samples from screening (Kavanagh et al, 2013), and the monitoring of HPV-associated disease through national screening and colposcopy services. Preliminary analysis of HPV prevalence in the Scottish catch-up cohort corroborates previous studies (Paavonen et al, 2009; Wheeler et al, 2012) and shows that the bivalent vaccine is strongly associated with a reduction in both HPV 16 and 18, while affording cross-protection against HPV 31, 33 and 45 (Kavanagh et al, 2014).

Our analysis focused on the risk of CIN 1, 2 and 3 dependent on the number of doses of vaccine received adjusted for cohort year, deprivation and age at observation. Cohort year is used to model the possible background patterns of changes in sociological behaviour that may exist from one birth cohort to the next. This may influence the levels of CIN 1, 2 and 3 observed, that is, those born in 1988 have two invitations to be screened and 3 years of opportunistic screening between those periods, while those people born in 1989 will not have the same screening opportunities, hence the reduction in all grades of CIN in the 1989 cohort. In the sensitivity analyses, which considered a more homogeneous population, there was no significant change in the numbers of CIN 1 and 2 by cohort year.

Although there was a significant reduction in all grades of CIN associated with 3 doses of vaccine in this cohort, no statistically significant reduction was observed in individuals who were partially immunised. However, almost all of the women who received two doses of vaccine in this cohort were immunised at

Table 3. Adjusted odds of CIN 1, 2 and 3 in the 6 months following the date of screening in those attending screening at age 20 or 21 who have 6 months of follow-up available

\begin{tabular}{|c|c|c|c|c|c|c|c|c|c|c|c|}
\hline & OR & $95 \% \mathrm{Cl}$ & $P$-value & & OR & $95 \% \mathrm{Cl}$ & $P$-value & & OR & $95 \% \mathrm{Cl}$ & $P$-value \\
\hline CIN 1 & & & & CIN 2 & & & & $\mathrm{CIN} 3$ & & & \\
\hline Unvaccinated & 1 & - & & Unvaccinated & 1 & - & & Unvaccinated & 1 & - & \\
\hline 1 Dose & 0.92 & $0.45,1.88$ & 0.8046 & & 0.64 & $0.28,1.45$ & 0.276 & & 1.91 & $1.11,3.29$ & 0.0193 \\
\hline 2 Doses & 0.66 & $0.36,1.20$ & 0.1765 & & 0.62 & $0.34,1.13$ & 0.1216 & & 0.75 & $0.41,1.36$ & 0.3524 \\
\hline 3 Doses & 0.59 & $0.44,0.79$ & 0.0006 & & 0.4 & $0.29,0.55$ & $<0.0001$ & & 0.5 & $0.36,0.69$ & $<0.0001$ \\
\hline 1988 & 1 & - & & & 1 & - & & & 1 & - & \\
\hline 1989 & 1 & $0.80,1.25$ & 0.9760 & & 0.89 & $0.72,1.11$ & 0.2976 & & 0.78 & $0.62,0.98$ & 0.0332 \\
\hline 1990 & 0.91 & $0.72,1.16$ & 0.4567 & & 0.85 & $0.68,1.06$ & 0.1552 & & 0.92 & $0.74,1.15$ & 0.4744 \\
\hline 1991 & 1.04 & $0.77,1.39$ & 0.8160 & & 0.98 & $0.73,1.31$ & 0.8894 & & 0.85 & $0.63,1.15$ & 0.2807 \\
\hline 1992 & 1.14 & $0.78,1.67$ & 0.4737 & & 0.79 & $0.52,1.21$ & 0.2809 & & 0.56 & $0.35,0.89$ & 0.0143 \\
\hline SIMD 1 & 1 & - & & & 1 & - & & & 1 & - & \\
\hline SIMD 2 & 0.65 & $0.51,0.83$ & 0.0005 & & 0.77 & $0.62,0.95$ & 0.0183 & & 0.83 & $0.66,1.03$ & 0.095 \\
\hline SIMD 3 & 0.71 & $0.56,0.91$ & 0.0074 & & 0.53 & $0.41,0.68$ & $<0.0001$ & & 0.6 & $0.47,0.78$ & $<0.0001$ \\
\hline SIMD 4 & 0.74 & $0.58,0.94$ & 0.0181 & & 0.57 & $0.44,0.73$ & $<0.0001$ & & 0.59 & $0.46,0.77$ & $<0.0001$ \\
\hline SIMD 5 & 0.66 & $0.52,0.84$ & 0.0013 & & 0.37 & $0.28,0.49$ & $<0.0001$ & & 0.33 & $0.24,0.46$ & $<0.0001$ \\
\hline
\end{tabular}

Abbreviations: $\mathrm{Cl}=$ confidence interval; $\mathrm{CIN}=$ cervical intraepithelial neoplasia; $\mathrm{OR}=$ odds ratio; $\mathrm{SIMD}=\mathrm{Scottish}$ Index of Multiple Deprivation .

Bold indicates these variables being statistically significant, that is, $P<0.05$. 
0 and 1 month. Further data are required to assess what protective effect is afforded by $<3$ doses of vaccine since only $3.8 \%$ of women in our cohort were partially vaccinated. We hope to elucidate the long-term efficacy of a two-dose vaccine regimen through the analysis of updated quarterly colposcopy extracts to the national surveillance programme since studies suggest a two-dose regimen may be both protective and cost-effective (Kreimer et al, 2011; Romanowski et al, 2011).

HPV vaccination and regular cervical screening offer the best combination for prevention of cervical cancer. However, knowledge and awareness of HPV infection, cervical cancer and screening in young girls who have been vaccinated against the virus, is surprisingly low (Bowyer et al, 2013). This has prompted concerns that those girls who have been vaccinated may not realise they still require regular cervical screening (Marlow et al, 2007; Henderson et al, 2011). Reassuringly, we found that there was no reduction in the initial uptake of cervical screening in those women who were born in 1990 and had been vaccinated in the catch-up cohort. Nevertheless, it is imperative that health education initiatives continue to emphasise the importance of attendance at cervical screening for vaccinated women. This is especially important, given the association of increased levels of HPV positivity and poor attendance at cervical screening with increased deprivation (O'Leary et al, 2011; Baker and Middleton, 2003).

One of the main limitations with attributing reductions in CIN to vaccination is that women born in 1991-1992 who were fully vaccinated, would likely have been at school up to the age of 18 , while the majority of the unvaccinated women in these cohorts would likely have left school at age 16 . Coupled with this is the observation from a baseline prevalence study prior to vaccination that the levels of HPV positivity among girls aged 16-18 who had left school was much higher compared to those who were still at school (O'Leary et al, 2011). Thus the comparison of fully vaccinated with unvaccinated cohorts is confounded with leaving school, but no individual adjustment can be made for this with the data available in this study. Attributing the reduction in severe disease solely to vaccination will overestimate the impact of the vaccination but given that this study reports a 55\% reduction in the relative risk of CIN 3 associated with three doses of vaccine, it is extremely unlikely that all of this effect is confounded with leaving school early.

Our work builds upon previous studies that assessed high-grade abnormalities and CIN data in both vaccinated and unvaccinated individuals, respectively (Powell et al, 2012; Kavanagh et al, 2014; Crowe et al, 2014; Baldur-Felskov et al, 2014). To our knowledge, this is the first study to report vaccine effectiveness findings against cervical lesions for the bivalent vaccine in a population rather than in a trial setting. The strengths of our analyses are that we have a large, complete population-based dataset on cervical screening that we can then directly link to disease and vaccination status through use of our national databases. Scotland is therefore in a strong position to assess the ongoing impact of the HPV vaccine on HPV-associated disease in the years ahead, including assessment in 2015 of vaccine impact in the routinely immunised girls. These data are generalisable to countries that have achieved high HPV vaccine uptake such as Australia, Portugal and Rwanda (Hopkins and Wood, 2013). This study highlights the gains that can be achieved if action is taken to overcome recognised barriers to high vaccine uptake.

\section{ACKNOWLEDGEMENTS}

Monitoring and evaluation of the HPV immunisation programme in Scotland is funded by the Scottish Government. This research is partially funded by the CSO grant, CZH/4/528. We thank Dr Katy Sinka for her invaluable contribution in setting up the HPV surveillance programme.

\section{CONFLICT OF INTEREST}

The authors declare no conflict of interest.

\section{REFERENCES}

Baker D, Middleton E (2003) Cervical screening and health inequality in England in the 1990s. J Epidemiol Community Health 57: 417-423.

Baldur-Felskov B, Dehlendorff C, Munk C, Kjaer SK (2014) Early impact of human papillomavirus vaccination on cervical neoplasia-nationwide follow-up of young Danish women. J Natl Cancer Inst 106: djt460.

Bhopal RS, Bansal N, Steiner M, Brewster DH. Scottish Health and Ethnicity Linkage Study (2012) Does the 'Scottish effect' apply to all ethnic groups? All-cancer, lung, colorectal, breast and prostate cancer in the Scottish Health and Ethnicity Linkage Cohort Study. BMJ Open 2: pii: e001957.

Bowyer HL, Marlow LA, Hibbitts S, Pollock KG, Waller J (2013) Knowledge and awareness of HPV and the HPV vaccine among young women in the first routinely vaccinated cohort in England. Vaccine 31: 1051-1056.

Brotherton JML, Fridman M, May CL, Chappell G, Saville AM, Gertig DM (2011) Early effect of the HPV vaccination programme on cervical abnormalities in Victoria, Australia: an ecological study. Lancet 377: 2085-2092.

Cancer research UK (2010) London: Cancer Research UK. Cervical cancerUK incidence statistics http://info.cancerresearchuk.org/cancerstats/types/ cervix/incidence/. Accessed 07 February 2014.

Crowe E, Pandeya N, Brotherton JM, Dobson AJ, Kisely S, Lambert SB, Whiteman DC (2014) Effectiveness of quadrivalent human papillomavirus vaccine for the prevention of cervical abnormalities: case-control study nested within a population based screening programme in Australia. BMJ Open 348: g1458.

Cuschieri K, Brewster DH, Williams ARW, Millan D, Murray G, Nicoll S, Imrie J, Hardie A, Graham C, Cubie HA (2010) Distribution of HPV types associated with cervical cancers in Scotland and implications for the impact of HPV vaccines. Br J Cancer 102: 930-932.

Henderson L, Clements A, Damery S, Wilkinson C, Austoker J, Wilson S. HPV Core Messages Writing Group (2011) 'A false sense of security'? Understanding the role of the HPV vaccine on future cervical screening behaviour: a qualitative study of UK parents and girls of vaccination age. J Med Screen 18: 41-45.

Hopkins TG, Wood N (2013) Female human papillomavirus (HPV) vaccination: global uptake and the impact of attitudes. Vaccine 31 : 1673-1679.

Information Services Division, Scotland (2011) Estimate of HPV vaccine uptake in Scotland by year of birth http://www.isdscotland.org/HealthTopics/Child-Health/Publications/2011-09-22/HPV_by_YOB_Aug11. xls. Accessed 07 February 2014.

Information Services Division, Scotland (2012) Estimate of HPV vaccine uptake in Scotland by year of birth, catch-up cohort http://www. isdscotland.org/Health-Topics/Child-Health/Publications/2012-09-25/ HPV_Catch-up_Programme.xlsAccessed 07 February 2014.

Kavanagh K, Pollock KGJ, Potts A, Love J, Cuschieri K, Cubie H, Robertson C, Donaghy M (2014) Introduction and sustained high coverage of the HPV bivalent vaccine leads to a reduction in prevalence of HPV 16/18 and closely related HPV types. Br J Cancer 110: 2804-2811.

Kavanagh K, Sinka K, Cuschieri K, Love J, Potts A, Pollock KG, Cubie H, Donaghy M, Robertson C (2013) Estimation of HPV prevalence in young women in Scotland; monitoring of future vaccine impact. BMC Infect Dis 13: 519 .

Kreimer AR, Rodriguez AC, Hildesheim A, Herrero R, Porras C, Schiffman M, González P, Solomon D, Jiménez S, Schiller JT, Lowy DR, Quint W, Sherman ME, Schussler J, Wacholder S. CVT Vaccine Group (2011) Proof-of-principle evaluation of the efficacy of fewer than three doses of a bivalent HPV16/18 vaccine. J Natl Cancer Inst 103: 1444-1451.

Malagon T, Drolet M, Boily MC, Franco EL, Jit M, Brisson J, Brisson M (2012) Cross-protective efficacy of two human papillomavirus vaccines: a systematic review and meta-analysis. Lancet Infect Dis 12: 781-789.

Marlow LAV, Waller J, Wardle J (2007) Public awareness that HPV is a risk factor for cervical cancer. Br J Cancer 97: 691-694.

O’Leary MC, Sinka K, Robertson C, Cuschieri K, Lyman R, Lacey M, Potts A, Cubie HA, Donaghy M (2011) HPV type-specific prevalence using a urine assay in unvaccinated male and female 11- to 18-year olds in Scotland. Br J Cancer 104: 1221-1226. 
Paavonen J, Naud P, Salmeron J, Wheeler CM, Chow SN, Apter D, Kitchener H, Castellsague X, Teixeira JC, Skinner SR, Hedrick J, Jaisamrarn U, Limson G, Garland S, Szarewski A, Romanowski B, Aoki FY, Schwarz TF, Poppe WA, Bosch FX, Jenkins D, Hardt K, Zahaf T, Descamps D, Struyf F, Lehtinen M, Dubin G (2009) Efficacy of human papillomavirus (HPV)-16/18 AS04adjuvanted vaccine against cervical infection and precancer caused by oncogenic HPV types (PATRICIA): final analysis of a double-blind, randomised study in young women. Lancet 374: 301-314.

Powell SE, Hariri S, Steinau M, Bauer HM, Bennett NM, Bloch KC, Niccolai LM, Schafer S, Unger ER, Markowitz LE (2012) Impact of human papillomavirus (HPV) 16/18-related prevalence in precancerous cervical lesions. Vaccine 31: 109-113.

R Development Core Team (2014) R: A Language and Environment for Statistical computing. R Foundation for Statistical Computing: Vienna, Austria, ISBN 3-900051-07-0, URL http://www.R-project.org/.

Romanowski B, Schwarz TF, Ferguson LM, Peters K, Dionne M, Schulze K, Ramjattan B, Hillemanns P, Catteau G, Dobbelaere K, Schuind A, Descamps D (2011) Immunogenicity and safety of the HPV-16/18 AS04adjuvanted vaccine administered as a 2 -dose schedule compared with the licensed 3-dose schedule: results from a randomized study. Hum Vaccin 7: 1374-1386.

Scottish Government (2005) Scottish Neighbourhood Statistics Guide. http:// www.scotland.gov.uk/Publications/2005/02/20697/52626. Accessed online 07 February 2014.
Scottish Government. Scottish Index of Multiple Deprivation http://www. scotland.gov.uk/Topics/Statistics/SIMD. Accessed online 07 February 2014.

Sinka K, Kavanagh K, Gordon R, Love J, Potts A, Donaghy M, Robertson C (2014) Introduction, high and equitable coverage of adolescent HPV vaccine in Scotland. J Epidemiol Community Health 68: 57-63.

Smith JS, Lindsay L, Hoots B, Keys J, Franceschi S, Winer R, Clifford GM (2007) Human papillomavirus type distribution in invasive cervical cancer and high-grade cervical lesions: a meta-analysis update. Int J Cancer 21: 621-632.

Wheeler CM, Castellsaque X, Garland SM, Szarewski A, Paavonen J, Naud P, Salmerón J, Chow SN, Apter D, Kitchener H, Teixeira JC, Skinner SR, Jaisamrarn U, Limson G, Romanowski B, Aoki FY, Schwarz TF, Poppe WA, Bosch FX, Harper DM, Huh W, Hardt K, Zahaf T, Descamps D, Struyf F, Dubin G, Lehtinen M (2012) Cross-protective efficacy of HPV-16/18 AS04-adjuvanted vaccine against cervical infection and precancer caused by non-vaccine oncogenic HPV types: 4-year end-of-study analysis of the randomised, double-blind PATRICIA trial. Lancet Oncol 13: 100-110.

This work is published under the standard license to publish agreement. After 12 months the work will become freely available and the license terms will switch to a Creative Commons AttributionNonCommercial-Share Alike 3.0 Unported License. 